\title{
ESTUDO DE PERMEABILIDADE EM ROCHAS FISSURADAS E CARSTIFICADAS DA REGIÃO DE NEUE WELT, ÁUSTRIA
}

\author{
NORBERT FENZL*
}

\begin{abstract}
PERMEABILITY IN FISSURED AND KARSTIFICATED ROCKS. The main goal of this investigation was to search for a mathematical method to describe hydrodynamic conditions (permeability, filtration speed and hydraulic gradient) in karstificated and fissured rocks. The choosen method, based on the identification of fissure-groups mainly responsible for the karstification process, has been developped by Kiraly in the Jurassic formations of France and Switzerland. In the present work, this method has been applied in karst-areas $40 \mathrm{~km}$ - SW of Viena, Austria.
\end{abstract}

Keywords: Permeability, fitration speed, hydraulic gradient, karst, fissured rocks.

\begin{abstract}
RESUMO O objetivo central das investigacões aqui apresentadas foi a busca de um método satisfatório para determinar as condições hidrodinâmicas (permeabilidade, velocidade de filtração e gradiente hidráulico) em rochas físsuradas e ojrsrificadas. A área escolhida para realizar a pesquisa, a chamada Neue Welt, é constituída por dois anticlinais de calcário triássico fortemente ramificados, a Serra da Hohe Wand e a Serra dos Fischauer Berge, situada a $40 \mathrm{~km}$ no sudoeste de Viena, Austria. Os dois anticlinais são separadas pela Bacia Sedimentar de Gosau. O método empregado se baseia em trabalhos desenvolvidos por Kiraly no jurássico suíço e francês e consiste na identificação das famílias de fissuras responsáveis para a formação do carst, na determinação do gradiente hidráulico e no cálculo do tensor de permeabilidade e da velocidade de filtração da formação geológica estudada.
\end{abstract}

Palavras-chaves: Permeabilidade, velocidade de filtração, gradiente hidráulico, carst, rochas fissuradas.

INTRODUÇÃO A área de estudo é situada na margem S W da Bacia de Viena, distante $40 \mathrm{~km}$ da capital, aproximadamente.

Ela é constutuída principalmente por dois anticlinais de calcário triássico fortemente carsficado - a Serra da Hohe Wand e a Serra dos Fischauer Berge, separadas por uma bacia sedimentar composta por depósitos de margas, argilas e arenitos da época Dan - Paleocênica. A Serra da Hohe Wand está situada na margem NW da bacia e constitui a principal fonte de abastecimento de águas subterrâneas da bacia, cuja atividade básica é a agricultura intensiva (Fig. 1).

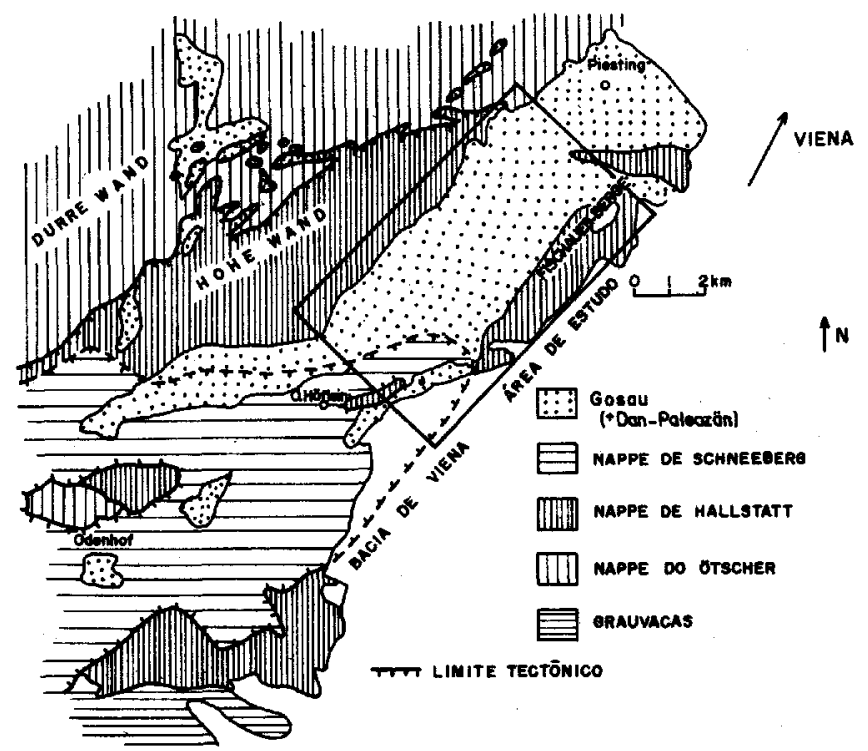

Figura 1 - Geologia e estrutura tectônica da área Figure 1-Geology and tectonic structure of the area
Por outro lado, a Serra dos Fischauer Berge, situada na margem SE da bacia numa posição topogrảfica inferior, funciona como "sistema de drenagem" da Bacia de Gosau. Para poder avaliar as entradas e saídas de águas subterrâneas da bacia, tornou-se necessária a determinação das condições hidrodinâmicas das formações geológicas de ambas as serras.

O objetivo principal da pesquisa foi, portanto, a determinação da permeabilidade média $(\bar{K})$ e da velocidade de filtração $(\bar{q})$ média para os dois anticlinais.

A metodologia empregada nesta pesquisa consiste em vários passos baseados nos trabalhos de Kiraly (1968, e 1971), que desenvolveu o método com sucesso no jurássico francês, e inclui:

a. Levantamento cartográfico de 50 grutas acessíveis. Todos os segmentos destas grutas são projetadas num plano horizontal, eliminando inclinações superiores a $50^{\circ}$. Mostrou-se que, em $70 \%$ dos casos, as inclinações não superam os $10^{\circ}$.

b. Levantamento superficial dos principais sistemas de fissuras da rocha calcária. Para esta finalidade foram escolhidos quatro pontos em cada serra, nos quais foram registradas todas as fissuras que cruzam uma linha horizontal de $10 \mathrm{~m}$ de comprimento. Durante o registro das fissuras foram distinguidos os seguintes aspectos: fissuras com uma extensão maior de $3 \mathrm{~m}$; fissuras abertas ou fechadas; fissuras que apresentam algum tipo de depósito ou estriações nas faces internas; e fissuras condutoras de água.

Os dados obtidos são projetados em diagramas que permitem a classificação precisa dessas manifestaçôes tectônicas.

c. Os resultados de (a) e (b) são comparados para identificar os sistemas de físsuras responsáveis pela formação dos elementos que constituem a parte essencial do carst. 
d. Cálculo da permeabilidade $\vec{K}_{i}$ (na sua forma vetorial) por meio da relação:

$$
K i=\frac{g}{12 \cdot \delta \cdot f_{i} \cdot d_{i}^{3} \cdot[\bar{I}-(\vec{n} \cdot \vec{n})]}
$$

$\mathrm{g}=9,81 \cdot \mathrm{m} / \mathrm{s}$

$\delta$ = viscosidade da água

$f=$ freqüência dos sistemas de fissuras registrados

$d=$ abertura média dos sistemas de fissuras

$\overline{\mathrm{I}}=$ a matriz identidade

$\mathbf{n}=\mathbf{o}$ vetor perpendicular à direção geral do grupo de fissuras consideradas. Este vetor é dirigido para o centro da esfera de projeção horizontal e representa vetorialmente o grupo de físsuras.

$$
\bar{I}=\left[\begin{array}{lll}
1 & 0 & 0 \\
0 & 1 & 0 \\
0 & 0 & 1
\end{array}\right]
$$

e

$$
\vec{n}=\left[\begin{array}{l}
x \\
y \\
z
\end{array}\right]
$$

e. Cálculo do gradiente $\left(J_{i}\right)$, na sua forma vetorial.

$\vec{J}_{i}=\left[\begin{array}{l}X_{i} \\ Y_{i} \\ Z_{i}\end{array}\right]$

f. Cálculo do vetor e dos valores absolutos da velocidade de filtração $(q)$.

$q i=\vec{K}_{i} \cdot \overrightarrow{J_{i}}$

Nas páginas seguintes reproduzimos somente os resultados mais importantes que interessam diretamente ao tema tratado, ou seja, a determinação de parâmetros hidrodinâmicos em regiõ es carsrificadas.

\section{BALANÇO HÍDRICO DA ÁREA Precipitação}

A Bacia de Gosau pertence a uma das regiões relativamente pobres em precipitações dos Alpes austríacos. A média anual calculada para o período de 1905 a 1950 é de $762 \mathrm{~mm}$.

A cobertura de neve não é contínua durante os meses de inverno, tanto decorrente de um microclima relativamente suave em comparação a outras regiões de mesma altitude, quanto decorrente de uma forte atividade dos ventos, que provocam uma distribuição desigual da espessura da neve.

A precipitação média anual entre 1968 e 1975 foi calculada em relação a Bacia de Gosau e da parte da mesa da Hohe Wand, situada numa região mais úmida e elevada e em grande parte responsável pelo abastecimento de águas subterrâneas da bacia como um todo.

$$
N=973 \mathrm{~mm} / \mathrm{a}
$$

Isto significa, relativo à superfície da área estudada, uma quantidade de água de

$N=5,351 \cdot 10^{6} \mathrm{~m}^{3} / a$

Assim, obtivemos para a Hohe Wand uma precipitação de

Este volume de água provém, portanto, da mesa (altiplano) da Serra da Hohe Wand e alimenta a Bacia de Gosau.
A precipitação diretamente incidente na bacia era, durante o mesmo período, de

$n=779 \mathrm{~mm} / \mathrm{a}$

\section{O que corresponde a um volume de água de}

$n=27,171 \cdot 10^{6} \mathrm{~m}^{3} / a$

Evapotranspiração A evapotranspiração (ET) da área investigada foi deduzida por interpelação das medidas feitas em três estações da região, calculadas através da relação de Turc (1954).

Para relacionar a evaporação geral da área, que inclui grandes partes sem cobertura vegetal permanente, a bacia de Gosau foi dividida em três zonas, de acordo com a permeabilidade dos solos: $50 \%$ da superfície da área pode ser considerada de permeabilidade boa; $20 \%$ da superfície pode ser considerada como mediamente permeáveis; e 30\% são praticamente impermeáveis, sendo que as precipitações são acumuladas em pequenos açudes e/ou escoam rapidamente para áreas mais permeáveis e/ou evaporam durante o caminho.

Tomando em conta a relação infiltração/evaporação, foi possível calcular a ET real para toda a bacia.

$E T=14,677 \cdot 10^{6} \mathrm{~m}^{3} / a$

Drenagem superficial e balanço geral Geograficamente, a Bacia de Gosau apresenta a vantagem importante de ser drenada somente por um único rio. Assim, o escoamento superficial da bacia pode ser determinado com bastante precisão e não requer nenhuma técnica sofisticada.

As medidas de escoamento superficial $\left(E_{s}\right)$ foram feitas num ponto estratégico, na saída da bacia, debaixo de uma ponte, que garantia um perfil geometricamente definido, facilitando o cálculo da velocidade do fluxo de água.

$\mathrm{O}$ valor médio do escoamento, baseado em medidas feitas quase diariamente durante o ano de 1975, foi:

$E_{\mathrm{s}}=6,464 \cdot 10^{6} \mathrm{~m}^{3} / \mathrm{a}$

É importante notar, que medidas posteriores mostram que este valor é bastante representativo, mesmo para períodos de vários anos.

A partir desses dados, foi determinado o balanço hídrico global da área. Sem entrar nos detalhes do procedimento, foram encontrados os seguintes valores:

$100 \%(\mathrm{P})=30 \%(\mathrm{I})+20 \%\left(\mathrm{E}_{\mathrm{s}}\right)+50 \%(\mathrm{ET})$

$\mathrm{P}=$ Precipitação

I = Infiltração

Os dados hidrológicos aqui apresentados não são objeto deste trabalho, devem servir somente de parâmetros para situar o leitor nas condições" hidrometeorológicas gerais da região.

HIDROGEOLOGIA Metodologia Como já foi mencionado anteriormente, a Bacia de Gosau está situada entre duas anticlinais de formações calcárias triássicas. Ambas apresentam uma carsrificação intensa (formação de mais de 100 grutas, das quais aproximadamente 70 acessíveis), fato de maior importância para a hidrogeologia da região.

A carsfificação é um processo anisotrópico e a forma e direção dos elementos de carst dependem basicamente de três fatores: a. da direção dos principais sistemas de fissuras; 
b. da permeabilidade geométrica desses sistemas; c. e do sentido principal do gradiente hidráulico.

Os trabalhos de Kiraly (1968) mostram que as formações cársticas superficiais muitas vezes não estão relacionadas com as estruturas das cavidades subterrâneas; que a carsúficação não depende do tipo de fissuras - tais como fissuras de stress ou strain - senão, principalmente, da relação entre gradiente hidráulico e direção espacial da físsura.

A projeção estereográfica, que relaciona todos os grupos de fissuras com os elementos estruturais do carst, permite estabelecer a relação entre ambos.

$\mathrm{O}$ primeiro passo consiste, portanto, num levantamento preciso de todos os grupos (ou famílias) de fissuras que aparecem na formação geológica considerada. E importante que realmente todos os grupos, mesmo aqueles que superficialmente possam aparecer como insignificantes, sejam registrados com precisão.

Em seguida, realiza-se um levantamento cartográfico de todas as cavernas acessíveis e projeta-se os elementos estruturais que apresentam inclinações inferiores a $50^{\circ}$ num diagrama. A figura 2 a mostra o exemplo de uma gruta situada na Serra dos Fischauer Berge.

Numa primeira aproximação, comparam-se as projeções dos grupos de fissuras e dos grupos de elementos cársticos.

No segundo passo, é necessário calcular ou determinar experimentalmente o gradiente hidráulico (i) das águas subterrâneas circulando na formação geológica considerada.

Finalmente, uma vez definida a relação entre carstificação, fissuramento e gradiente hidráulico, é possível cal-
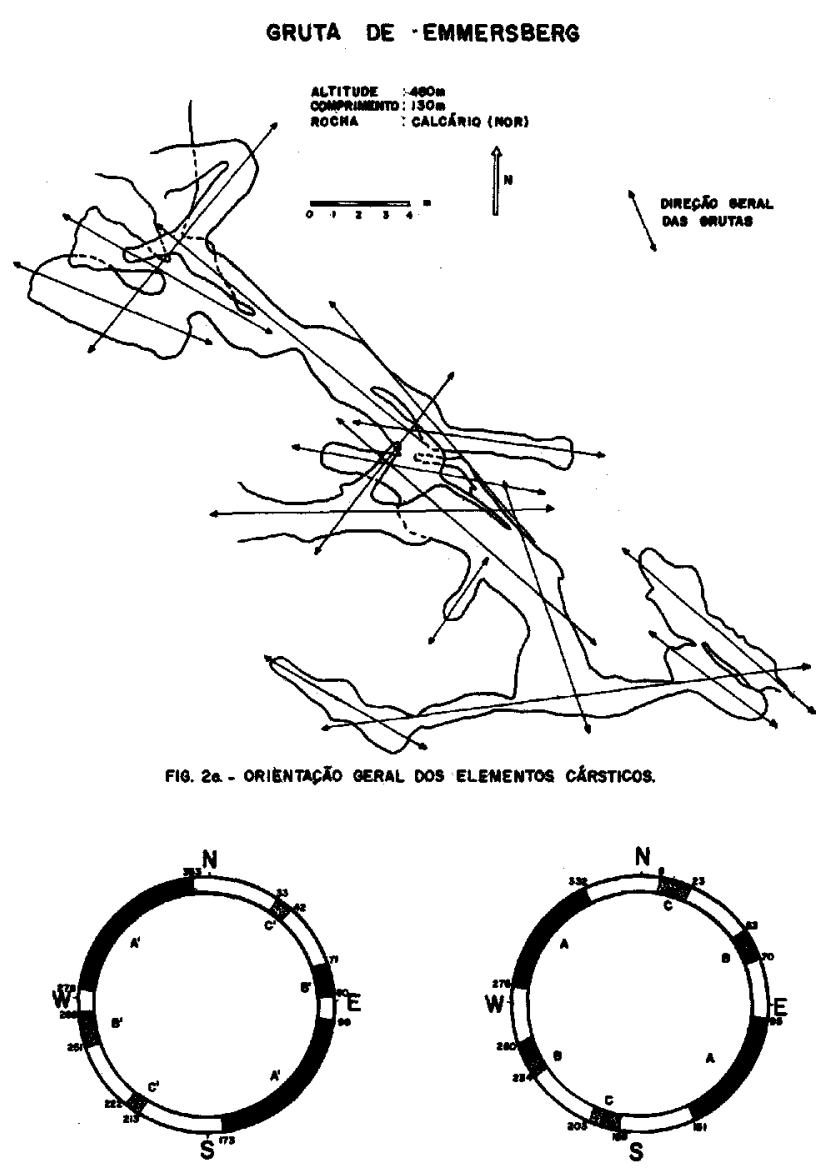

Figura 2 - a. Orientação geral dos elementos cársticos; b e c. Projeção e agrupamento dos elementos cársticos(b) Home Wand e (C Fischauer Berge

Figure 2 - a. General direction of karst-elements; b and c. Projection and separation of different groups of karst-elements cular a permeabilidade média da área ramificada $(\mathrm{K})$, assim como a velocidade média do fluxo de água nos grupos de fissuras registrados.

Cars/ificação e sistemas de fissuras Para este levantamento dos sistemas cársticos foram utilizados 50 planos de cadastro de grutas da área. Todos os elementos razoavelmente retos foram projetados num diagrama (Fig. 2b.c).

Os diagramas mostram uma congruência muito grande entre a ramificação das duas serras estudadas.

Em princípio, foi constatado que não há relações entre um determinado sistema de fissuras e o comprimento das diversas ramificações das grutas.

$\mathrm{O}$ volume (e espaço) ramificado depende da agressividade da água, da composição química da rocha, mas depende sobretudo do ângulo entre a fissura e o gradiente hidráulico (Fenzl 1977).

Assim, comparando as projeções dos grupos de fissuras e os elementos ramificados constatamos que os grupos de fissuras menos representativos são responsáveis pela maior rarsrificação e formação de grutas da região. Este aparente paradoxo se explica facilmente se observarmos a direção geral do gradiente hidráulico em ambas as serras: as fissuras são exatamente aquelas do tipo "stress", praticamente paralelas à direção geral do gradiente hidráulico.

Cálculo do tensor de permeabilidade A permeabilidade de um corpo fissurado pode ser determinado por meio do cálculo da permeabilidade de cada grupo de fissuras separadamente e da somatória de todas:

$\vec{K}=\epsilon \cdot K_{i}$

Segundo a lei básica de Darcy:

$\vec{q}=\bar{K} \cdot \vec{J}$

Onde:

$\vec{q}=$ vetor da velocidade de filtração

$K=$ tensor de permeabilidade geométrica

$\vec{J}=$ vetor do gradiente hidráulico

Para podermos aplicar o método aqui apresentado é necessário definir a seguinte hipótese de trabalho: que a água circula em fissuras com superfícies planas, de inclinação e direção variada, com um fluxo contínuo; que a transmissibilidade hidráulica seja isotrópica; que o corpo geológico estudado seja saturado.

Segundo Wittke \& Louis (1968), a velocidade de filtração

$\vec{q} i=\left[\frac{\left(g \cdot d^{3}\right)}{12 \cdot \gamma}\right] \cdot \overrightarrow{J p}$

Onde:

$g=9,81 \mathrm{~m} / \mathrm{s}$

$\gamma=$ viscosidade da água

$\overrightarrow{J p}=$ gradiente hidráulico definido pela direção da fissura $d=$ diâmetro (abertura) da fissura

Generalizando, pode-se afirmar que:

$\overrightarrow{J p}=A . \vec{J}$

Onde J é o gradiente hidráulico de toda a área considerada.

numa determinada fissura é igual a: 


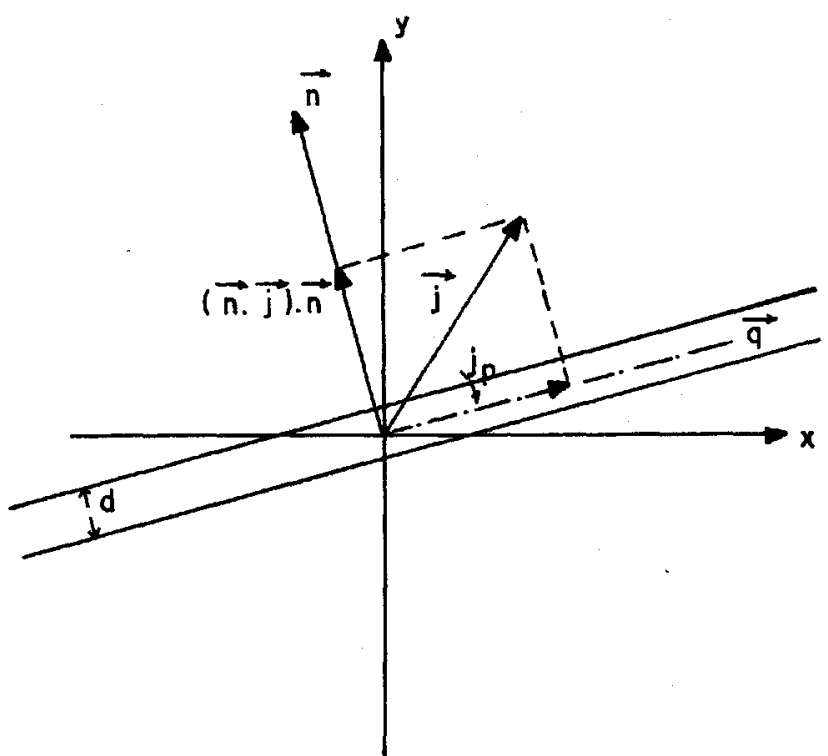

Figura 3 - Esquema geométrico do procedimento matemático

Figure 3 - Geometric proceedings of the method

Sendo (I) a matriz identidade e $(\vec{n})$ o vetor perpendicular à superfície da fissura (Fig. 3):

$\widetilde{I}=\left[\begin{array}{lll}1 & 0 & 0 \\ 0 & 1 & 0 \\ 0 & 0 & 1\end{array}\right]$

e

$\vec{n}=\left[\begin{array}{l}x \\ y \\ z\end{array}\right]$

Assim, $\overrightarrow{J p}=[\bar{I}-(\vec{n} \cdot \vec{n})] . \vec{J}$ sendo $\bar{A}=\bar{I}-(\vec{n} \cdot \vec{n})$

$\bar{A}$ é uma matriz geométrica de valor:

$\bar{A}=\left[\begin{array}{ccc}1-x & -z y & -x z \\ -y x & 1-y & -y z \\ -z z & -z y & 1-z\end{array}\right]$ mos:

Transferindo esta expressão para a equação (15), tere-

$\vec{q}=\left(\frac{g}{12 \lambda}\right) \cdot d^{3} \cdot A J$

Se considerarmos que o grupo de fissuras seja composto por (f) elementos por metro numa determinada direção, então:

$\bar{K}=\left(\frac{g}{12 \lambda}\right) \cdot f \cdot d^{3} \cdot \bar{A}$

Esta equação permite calcular a permeabilidade para cada grupo de fissuras separadamente. Posteriormente, aplicando a relação (13), é possível determinar a permeabilidade para todo o corpo geológico considerado.

Aplicação prática Partindo dos resultados do levantamento microtectônico e da carsrificação, um programa de computação, especialmente desenvolvido por Kiraly (1969) determina os valores de (f) e de (n). Os valores de (d) são tomados como abertura média de todas as fissuras registradas.

No caso específico das duas serras, foram escolhidas quatro estações nos dois casos. A Serra da Hohe Wand apresenta uma abertura média das fissuras responsáveis pela ozrsfificação igual a $d l=0,2 \mathrm{~mm}$ e no caso da Serra dos Fischauer Berge, $d i=0,3 \mathrm{~mm}$.

$A$ partir desses dados, podemos determinar a permeabilidade $(\bar{K})$ relativa a cada estação, sob forma tensorial.

Para simplificar, não serão produzidos aqui os resultados de cada estação individualmente, mas somente o valor da súmula tensorial de todos os quatro pontos de medida. Este valor representa a média global da permeabilidade relativa a cada área de estudo.

$\overline{\mathbf{K}}_{I}=\left[\begin{array}{ccc}150,13 & -50,358 & 389,392 \\ -50,358 & 1219,624 & 233,664 \\ 389,392 & 233,664 & 1977,015\end{array}\right] \mathrm{m} / \mathrm{s}$

\section{- Serra dos Fischauer Berge}

$\overline{\mathrm{K}}_{\mathbf{2}}=\left[\begin{array}{ccc}1641,662 & 685,128 & 201,676 \\ 685,128 & 1909,443 & -211,589 \\ 389,392 & 233,664 & 1977,015\end{array}\right] \mathrm{m} / \mathrm{s}$

- Serra da Hohe Wand

Cálculo do gradiente hidráulico Em seguida, foram determinados os gradientes hidráulicos gerais para a Serra da Hohe Wand $\left(\vec{J}_{1}\right)$ e a Serra dos Fischauer Berge $\left(\vec{J}_{2}\right)$. $\mathrm{O}$ gradiente hidráulico deve ser representado enquanto vetor no espaço tridimensional por meio de suas coordenadas. $\mathrm{O}$ sistema de coordenadas usado é ortonormado, com os eixos $(\mathrm{y})$ e $(x)$ representando as direções geográficas $\mathrm{N}-\mathrm{S}$ e $\mathrm{E}-\mathrm{W}$, respectivamente. $\mathrm{O}$ vetor unitário é representado por $O A$; o $\begin{array}{llll}\text { eixo } & (z) & \text { sendo } & \text { vertical }\end{array}$

Conhecendo-se:

- o ângulo do gradiente em relação à direção Norte - Sul;

- a inclinação do gradiente em relação à horizontal.

Ambas as informações são deduzidas de observações de campo. Projetando-se $\left(\vec{J}_{1}\right)$ no plano $(y-\mathrm{O}-x)$ e designando

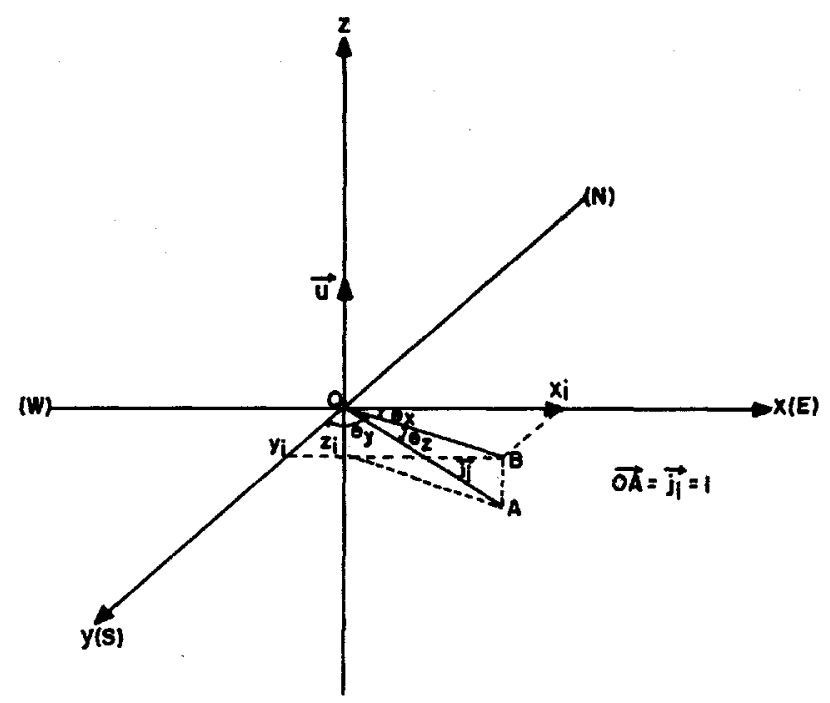

Figura 4 -Apresentação vetorial do gradiente hidráulico Figure 4 - Vectorial representation of the hydraulic gradient 
por (ao) o ângulo de $x$ - O - B, por $\left(a_{1}\right)$ o ângulo $y$ - O - B e por $(a z)$ o ângulo $\mathrm{A}-\mathrm{O}$ - B. As coordenadas do gradiente $(\mathrm{J}$; são, portanto, iguais a:

$\overrightarrow{J_{l}}=\left[\begin{array}{l}x_{i}=\cos \cdot a_{0} \\ x_{i}=\cos \cdot a_{0} \\ x_{i}=\cos . a_{0}\end{array}\right]$

Colocando-se nesta relação geral os dados relativos às áreas estudadas, obtém-se:

$$
\begin{gathered}
\overrightarrow{J_{I}}=\left[\begin{array}{rr}
x_{i}= & 0.80 \\
x_{i}= & 0.58 \\
x_{i}= & -0.03
\end{array}\right] \\
\mathrm{e} \\
\overrightarrow{J_{I}}=\left[\begin{array}{rr}
x_{i}= & 0.86 \\
x_{i}= & 0.49 \\
x_{i}= & -0.03
\end{array}\right]
\end{gathered}
$$

\section{Cálculo do vetor de velocidade de filtração}

acordo com as equações (14), (19) e (20) pode-se obter o valor da velocidade de filtração, multiplicando a permea-

Seja $\bar{K}=10^{-6} . A$ (para $A$ ver equação 2.3 .5$)$

$\overrightarrow{J_{I}}=\left[\begin{array}{c}X \\ Y \\ Z\end{array}\right]$

Assim,

$\vec{K} \cdot \vec{J}=10^{6}\left[\begin{array}{l}A=X\left(1-z y^{2}\right)-Y(y z)-Z(z x) \\ B=-X(y z)+Y\left(I-y^{2}\right)-Z(z y) \\ C=-X(z x)-Y(z y)+Z\left(1-z^{2}\right)\end{array}\right]$

$O$ valor absoluto de $\left[q_{3}\right]$ é igual a:

$\left[q_{3}\right]=\sqrt{A^{2}+B^{2}+C^{2}}$
- Com esta relação, é possível calcular o valor absoluto do vetor da velocidade de fíltração, tanto para um único grupo de fissuras, como para todo um corpo geológico. Aplicado à nossa área de estudo, obtemos:

- Serra da Hohe Wand

- Velocidade de filtração média vetorial

$\overrightarrow{q_{1}}=10^{-6}\left[\begin{array}{r}1223,655 \\ 660,086 \\ 387,726\end{array}\right] \mathrm{m} / \mathrm{s}$

- Velocidade de filtração, valor absoluto

$\left[\mathrm{q}_{1}\right]=1,443 \cdot 10^{-3} \mathrm{~m} / \mathrm{s}$

- Serra dos Fischauer Berge

- Velocidade de filtração média vetorial

$\vec{q}_{1}=10^{-6}\left[\begin{array}{l}1740,922 \\ 1009,943 \\ -15,833\end{array}\right] \quad \mathrm{m} / \mathrm{s}$

- Velocidade de filtração, valor absoluto

$\left[\mathrm{q}_{2}\right]=2.012 \cdot 10^{-3} \mathrm{~m} / \mathrm{s}$

CONCLUSÃO O método empregado mostrou ter três vantagens importantes: a. a simplicidade do levantamento dos grupos de fissuras; b. a precisão dos valores obtidos. Assim, a velocidade de filtração foi comprovada em ocasiões posteriores com métodos isotópicos (Fenzl 1977); c. os baixos custos operacionais do método.

A desvantagem consiste basicamente no fato de que o método exige o levantamento cartográfico de pelo menos uma gruta representativa da área. Em regiões com grandes dificuldades de acesso às cavernas cársticas, esse método pode ser inviabilizado.

bilidade pelo gradiente hidráulico correspondente.

\section{REFERÊNCIAS BIBLIOGRÁFICAS}

FENZL, N. 1977. Hydrogeologische Studie dês Gebietes Hohe Wand und Fischauer Berge. Verh Geol. B., 2(A): 121-164.

KIRALY, L. 1969. Anisotropie et héterogénité de Ia perméabilité dans lês calcaires fissures. Eclogae Geol. Helv., 62:613-619.

KIRALY, L.; MATHEY, B.; TRIPET, J. P. 1971. Fissuration et orientation dês cavités souterraines. Region de Ia Gotte de Milandre (Jura tabulare). Buli. soe. Neuchatel. Sei. nat., 94:99-114.

KIRALY. L. 1968. Elements structuraux et alignements dês phénoménes karstiques (Region Gouffre du Petit - Pré, St. Livres, Jura Vaudois). Buli. soe. Neuchatel. Sei. nat., 91:127-146.
TURC, L. 1954. Calcul du bilian de 1'eau. Evaluation en fonction dês precipitations et dês temperatures. Ass. Int. Hydrogeol. Soe. 38:188-202.

WITTKE, W. \& LOUIS, CL. 1968. Modellversuche zur Durchstroemung Klueftiger Medien. Felsmech. Ing. Geol., 4:52-78.

MANUSCRITO A792 Recebido em 22 de novembro de 1993 Revisão do autor em 13 de outubro de 1994 Revisão aceita em 23 de julho de 1994 